\title{
Optimal Design of Truss Structures based on the New Improved Ant Colony Optimization Algorithm
}

\author{
Li Shi-feng ${ }^{1, a}$, Zhang Zhuo-qun ${ }^{1, b}$, Li Hong-nan ${ }^{2, c}$ and Ren zongdong ${ }^{1, d}$ \\ ${ }^{1}$ State Nuclear Power Planning, Design \& Research Institute, Beijing, 100095, China; ${ }^{2}$ Faculty of \\ Infrastructure Engineering, Dalian University of Technology, Dalian 116024, China \\ alishifeng@snpdri.com, ${ }^{b}$ zhangzhuoqun_2006@163.com, ${ }^{c}$ hnli@dlut.edu.cn \\ renzongdong@snpdri.com,
}

\begin{abstract}
Keywords: Ant Colony Optimization (ACO); Traveling Salesman Problem (TSP); Truss Structure; Optimal Design; Discrete variable.
\end{abstract}

Abstract. This paper highlighted a new improved ant colony optimization algorithm was realized to deal with structures optimization problems efficiently, with several special improvements. The new algorithm procedure was written by the MATLAB language. The numerical simulation results demonstrated that this new methodology revealed more obvious advantage at truss structural optimization problems in discrete system. For the civil engineering designer, this new method is very simple and practical, and much more suitable.

\section{Introduction}

Truss structural optimization design is a critical and challenging activity that has received considerable attention in the last decades, because of its applicability in a wide range of practical design problems. In most engineering design activities, the design objective could be simply to minimize the cost of production or maximize the efficiency of production in minimal computational time and with minimal computer storage based on mathematical theory [1], while considering multiple loading conditions, stress and displacement constraints, minimum size limits on members and so on. In recent years, the swarm intelligence algorithm involving ant colony optimization, genetic algorithm, particle swarm optimization and so on, had been applied to the complex civil engineering successfully [2-3]. As a research hotspot of swarm intelligence, the ACO was a recently developed based on population approach and had been successfully applied in several NP-hard combinatorial optimization problems.

Although the ACO had a great progress in many aspects, it was not satisfied completely for project in civil engineering, especially for truss structure optimization problem. In this paper, a new improved algorithm was presented and had been applied to truss structures optimization problem successfully.

\section{Improved Ant Colony Optimization}

Since the first ant colony optimization had been proposed by Dorigo in Italy, this algorithmic method had attracted a lot of researchers and had a significant level of maturity, included on parallel distributed computing, great research capability, and strong adaptability and so on[4-6]. As a research hotspot, it had made a great progress in performance, application field and optimum result. The improved ant colony optimization algorithm mainly included the following categories: Ant-Q System[7], MAX-MIN Ant System[8], Adaptive ACO[9] and so on.

\section{The New Algorithm System}

In the traveling salesman problem, a set of cities are given and the distance between each of them is known. The goal is to find the shortest tour that allows each city to be visited once [4-6]. Its main characteristic is that the pheromone values are updated by all the $m$ ants that have built a solution itself in the iteration. The ant starts from a random city and iteratively moves to another city until its tour is completed and the ant should be back at the starting point. The new algorithm system has been improved as follow : 
(1). Model Recombination: The basic ant colony optimization, including ant-cycle, ant-density and ant-quantity model, was explained the differences in details by Dorigo, in1996. The researchers illustrated that the ant-cycle model optimized effect was the best. The mainly reason was that global information was taken and the searching ability and range was much stronger. The others only showed the local feature. In order to display different models' features, this paper recombined a new model with ant-cycle and ant-density model, named as $\mathrm{ACO}(\mathrm{C})+\mathrm{ACO}(\mathrm{D})$ model.

(2). Local Optimization: In civil engineering desgin, the local optimal result was not equal to worse result. Selecting and exploiting the local optimum sufficiently was very indispensable. After the each iteration, the ants will look for the local optimization route in the defined region by ant-density model until meet the suspensive condition, in this paper. If the local optimization route is better than this iteration global optimum, it will be consider as substitution.

(3). Local Ant Database: The outstanding elements can be found or hided in anyone result, and can be considered as the impotent factor to develop convergence performance. In this article, the local ant database is built by these different sections, and these sections must be recorded by display frequency. The local ant database also needs to be updated after each search process. The excellent local sections not only provide the information of the "unimpeded section" and "block section" in the next local or global search, but also obviously reduce the frequency of seeking numerous similar results in this algorithm.

(4). Specific Definition: After the ants finish a normal global optimization by ant-cycle model, the algorithm procedure will enter into the local search part to find local result by ant-density model. The algorithm in the local search part was divided into two situations.1.If the program can construct a "special sections" after a global search process, the ants quickly seek the local optimal solution in "Scope 1"; 2. If a "special section" can not be constructed, the ants will hunt some undiscovered paths, and expand the colony's searching range and scale of the "local ant database" in "Scope 2".

The "Special Section" is considered as follow. After the each global iteration, the several same continuation visited cities usually can be found in the paths, whose length is shorter than the average level. The continuation visited cities can be defined as special section. The "Unimpeded Section" about continuation visited cities is conducive to find the best optimization in the local and global search progress and the ineffectiveness section is regarded as "Block Section". The "Unimpeded Section" and "Block Section" come from the local ant database and react against. The "Scope 1" is a local optimization region based on the special sections. In this scope, all the ants must visit these special cities one by one. To the contrary, the "Scope 2" means that the all ants can not visit these special cities which are several old special sections reported in the local ant database.

\section{The New Algorithm System}

In this section, the complete operation process of the new ACO algorithm was introduced and the well-known traveling salesman problem would be as benchmark. It was organized as follows:

Step 1. Initialize: Set time counter: $t=0$; set cycles: $N=0$; set the maximum number of cycles $N_{\max }$; place the $m$ ants on the n nodes. The intensity of trail at time $0, \tau_{i j}=$ const should be set to arbitrarily chosen small values and $\tau_{i j}=0$.

Step 2. Cycle number: $N \leftarrow N+1$.

Step 3. Initialize the tabu list: $k=1$.

Step 4. The number of ants: $k \leftarrow k+1$.

Step 5. Choose the town $\mathrm{j}$ to move with probability $P_{i j}$ and $j \in\left\{C-t a b u_{k}\right\}$.

Step 6. Move the $k$ - th ant to the town $j$, and insert town $j$ in $t a b u_{k}(s)$. Then, repeat the above until tabu list is full. $\{$ This step will be repeated $(n-1)$ times. $\}$.

Step 7. If $k<m$, the produce is going on the step 4. Otherwise it will run the step 8.

Step 8. Establish a "Local Ant Database" and seek some "Special Sections".

Step 9. If the program can construct a "Special Sections", it will go to the step 10, or to the step 11. 
Step 10. The ants will quickly find out the best local solution in "Scope 1" according to the principle of "Special Sections" and "Unimpeded Section".

Step 11. The step will expand the scale of "Local Ant Database", look for some undiscovered paths and compel the next global ants to far away from previous search region in "Scope 2" according to the principle of "Block Section".

Step 12. Update the pheromone.

Step 13. If $N<N_{\max }$ empty all tabu lists and go to step 2. In contrary, print shortest tour and stop it.

\section{Truss Structural Optimization}

Generally, the mathematical model that come from actual engineering design in structural optimization, is discontinuous, non-differentiable, multi-peak and so on, and many variables (for example: component size or section) are discrete. Additionally, it is approved that the ant colony optimization algorithm is better than other algorithms when solve truss structures cross-section optimization problems [10-11]. In order to express its better efficiency and suit for practical engineering, the paper presents that the new ACO algorithm is used to structural optimization problem in discrete system.

\section{5-Bar Truss Structure}

The Fig. 1 showed a 25-bar spatial truss structure. In this example, designs were performed for a multiple loading case. The material density $E=68.96 \mathrm{GPa}$, and $\rho=27150.8 \mathrm{~N} / \mathrm{m}^{3}$. The structural members of this truss were arranged into eight groups. This spatial truss was subjected to the two loading conditions, and the maximum displacement limitations of $\pm 8.89 \mathrm{~mm}$ were imposed on every node. The ranges of cross-sectional areas are $\{0.774,1.355,2.142,3.348,4.065,4.632,6.542,7.742$, $9.032,10.839,12.671,14.581,21.483,34.839,44.516,52.903,60.258,65.226\}\left(\mathrm{cm}^{2}\right)$. The control parameters of the present algorithm are given in Table.1. The other data can be detailed specifications by relevant references[12-16].

Table.1 Control Parameters of the new ACO algorithm

\begin{tabular}{cccccccc}
\hline Control Parameters & $\alpha$ & $\beta$ & $\rho$ & $m_{G}$ & $m_{L}$ & $N_{\max }$ \\
\hline Numerical Value & 1 & 0.2 & 0.1 & 100 & 50 & 10 & 200 \\
\hline
\end{tabular}

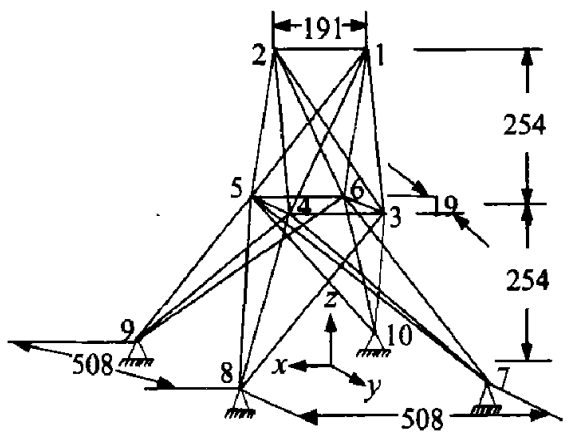

Fig.1 25-Bar Truss Structure Table.2 Comparison of Final Designs for 25-Bar Transmission Tower Truss

\begin{tabular}{ccccccccc}
\hline \multirow{2}{*}{ Number } & & \multicolumn{9}{c}{ Final cross-Sectional Areas } & $\left(\mathrm{cm}^{2}\right)$ & & \\
& Ref.12 & Ref.13 & Ref.14 & Ref.15 & Ref.16 & ACO & AS $_{\text {rank }}$ & This paper \\
\hline 1 & 0.0645 & 0.0645 & 0.1226 & 0.1032 & 0.0645 & 0.0645 & 0.0645 & 0.0645 \\
2 & 13.175 & 11.323 & 11.529 & 13.187 & 12.768 & 13.175 & 12.712 & 15.480 \\
3 & 19.362 & 18.510 & 19.400 & 18.264 & 19.426 & 19.362 & 19.713 & 15.480 \\
4 & 0.0645 & 0.0645 & 0.0645 & 0.0645 & 0.0645 & 0.0645 & 0.0645 & 0.0645 \\
5 & 0.0645 & 0.0645 & 0.0645 & 0.0645 & 0.0645 & 0.0645 & 0.0645 & 0.0645 \\
6 & 4.4132 & 5.4516 & 4.4710 & 4.3161 & 4.2387 & 4.4132 & 4.5474 & 5.1600 \\
7 & 10.485 & 12.974 & 12.155 & 11.058 & 10.826 & 10.485 & 10.711 & 10.970 \\
8 & 17.240 & 15.987 & 16.755 & 17.548 & 17.374 & 17.240 & 16.931 & 17.420 \\
Weight (N) & 2462.4 & 2423.6 & 2434.9 & 2426.8 & 2426.0 & 2425.4 & 2425.8 & 2410.6 \\
\hline
\end{tabular}

The optimization processes are illustrated in Fig.2, and the results are given in Table.2. This table showed a good correspondence, with previous results, of the design obtained by the new improved 
ACO method, and its efficiency. The comparison indicated that the method of this paper gave a better optimal design than those optimization algorithms in this procedure with a weight about $2.0 \%$ lower. In a word, it could be approved that the improved ant colony optimization model was very useful and success in truss structure.

\section{Conclusions}

This paper highlighted that the new improved ant colony optimization algorithm worked well in truss structural optimization problem by MATLAB system. The detail conclusions were as follows. This improved model, which was regarded as $\mathrm{ACO}(\mathrm{C})+\mathrm{ACO}(\mathrm{D})$ model with "Local Ant Database" and "Specific Definition", could find a good optimization result with convergence speed higher and search space wider. The numerical simulation results illustrated that the improved algorithm was obviously better than basic ACO and several better improved ACO algorithm. Therefore, this new improved algorithm have a good application prospect for the practical complexity engineering.

\section{Acknowledgments}

This work is supported by the National Natural Science Foundation of China under Grant no. 50638010 and the Fund for Creative Research Groups under Grant no. 51121005. This support is greatly appreciated.

\section{References}

[1] Qian, L.X. Optimum design of engineering structures. Beijing: Water Power Press, (1983).

[2] Engelbrecht, A.P. Fundamentals of computational swarm intelligence. Chichester, (2005).

[3] Dorigo, M. and Caro, G.D. Ant colony optimization: a new meta-heuristic. In D. Corne, M. Dorigo and F. Glover, (Eds.), New Ideas in Optimization, McGraw-Hill, (1999), pp-11-32.

[4] Dorigo, M., Maniezzo, V. and Colorni A. The ant system: optimization by a colony of cooperating agents. International Journal for IEEE Transactions on Systems, Man, and Cybernetics-Part B, 26, (1996), pp-29-42.

[5] Dorigo, M. and Gambardella, L.M. Ant colonies for the traveling salesman problem. International Journal for BioSystems, 43, (1997), pp-73-81.

[6] Duan, H.B. Ant Colony Algorithms: Theory and Application. Science Press, (2005), Beijing.

[7] Dorigo, M. and Gambardella, L.M. A study of some properties of Ant-Q. Proceedings of the 4th International Conference on Parallel Problem Solving from Nature, (1996), pp-656-665.

[8] Stützle, T. and Hoos, H.H. MAX-MIN Ant System. Future Generation Computer Systems. International Journal for Elsevier Science, 16(8), (2000), pp-889-914.

[9] Gao, S.W., Guo, L. Du, Y.Q. Yang, N. and Chen, L. Adaptive ant colony algorithm based on dynamic weighted rule. Journal for Computer Application. 27(7), (2007), pp-1741-1743.

[10]Camp, C. and Bichon, J. Design of space trusses using ant colony optimization. Structural Engineering, ASCE, 130 (5), (2004), pp-741-751.

[11] Kaveh, A. Azar, F.B. and Talatahari, S. Ant Colony Optimization for Design of Space Trusses. International Journal of Space Structures, 23(3), (2008), pp-167-181.

[12] Schmit, L.A. and Farshi, B. Some approximation concepts for structural synthesis. Journal of Amerizcan Institute of Aeronautics and Astronautics, 12(5), (1974), pp 231-233.

[13] Khan, M.R. Willmert, K.D. A New Optimality Criterion Method for Large Scale Structures. AIAA/ASME 19th Structures, Structural Dynamics and Materials Conference, (1978), pp 47-58.

[14] Tang, W.Y. Research and Application of Genetic Algorithm in Structural Optimization. Ph.D. Thesis, Dalian University of Technology, (2002), China.

[15] Gu, Y.X. Xiang, B.W. and Zhao, G.Z. Improved simulated annealing algorithm for cross. Section design optimization of truss structures. Chinese Journal of Computational Mechanics, 23(5), (2006), pp 546-552. 
[16]Li, L.J. Huang, Z.B. Liu, F. and Wu, Q.H. A heuristic particle swarm optimizer for optimization of pin connected structures. Computers and Structures, 85(7-8), (2007), pp 340-349. 